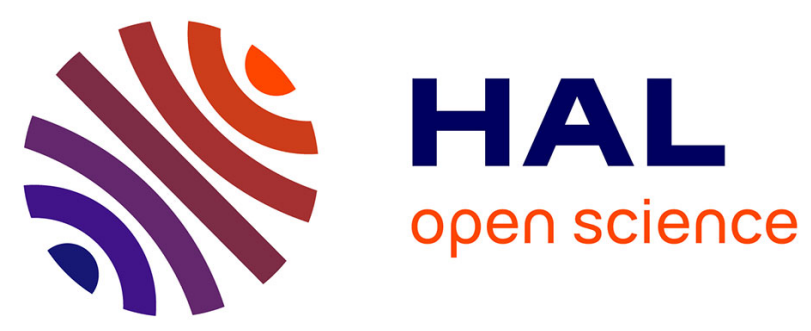

\title{
Contributions of the electronic spin and orbital current to the CoCl4(2-) magnetic field probed in polarised neutron diffraction experiments
}

Patrick Cassam-Chenaï, D. Jayatilaka

\section{- To cite this version:}

Patrick Cassam-Chenaï, D. Jayatilaka. Contributions of the electronic spin and orbital current to the CoCl4(2-) magnetic field probed in polarised neutron diffraction experiments. Journal of Chemical Physics, 2012, 137 (6), pp.064107. 10.1063/1.4737894 . hal-00687852v2

\section{HAL Id: hal-00687852 \\ https://hal.univ-cotedazur.fr/hal-00687852v2}

Submitted on 14 May 2012

HAL is a multi-disciplinary open access archive for the deposit and dissemination of scientific research documents, whether they are published or not. The documents may come from teaching and research institutions in France or abroad, or from public or private research centers.
L'archive ouverte pluridisciplinaire HAL, est destinée au dépôt et à la diffusion de documents scientifiques de niveau recherche, publiés ou non, émanant des établissements d'enseignement et de recherche français ou étrangers, des laboratoires publics ou privés. 


\title{
Contributions of the electronic spin and orbital current to the $\mathrm{CoCl}_{4}^{2-}$ magnetic field probed in polarised neutron diffraction experiments
}

\author{
Patrick Cassam-Chenaï \\ Laboratoire J. A. Dieudonné, UMR 6621 du CNRS, Faculté des Sciences, Parc \\ Valrose,06108 Nice cedex 2, France.cassam@unice.fr \\ Dylan Jayatilaka \\ School of Chemistry and Biochemistry, The University of Western Australia, 35 \\ Stirling Highway, CRAWLEY, WA 6009, Australia
}

\begin{abstract}
Polarised neutron diffraction experiments conducted at $4.2 \mathrm{~K}$ on $\mathrm{Cs}_{3} \mathrm{CoCl}_{5}$ crystals have been analysed by using a 4-dimensional model Hilbert space made of $a b$ initio $n$-electron wave functions of the $\mathrm{CoCl}_{4}^{2-}$ molecular ion. Two spin-orbit mixing coefficients and several configuration interaction coefficients have been optimized by fitting calculated magnetic structure factors to experimental ones, to obtain the best ensemble density operator that is representable in the model space. A goodness of fit, $\chi^{2}$, less then 1 has been obtained for the first time for the two experimental data sets available. In the present article, the optimized density operators are used to calculate the magnetic field densities that are the genuine observables probed in neutron diffraction experiments. Density maps of such observables are presented for the first time and numerical details are provided. The respective contributions of spin density and orbital current to the magnetic field density are analyzed.
\end{abstract}




\section{Introduction}

A molecule with a non zero spin density, such as an open-shell molecule in a magnetic field, produces a magnetic field density in space. If in addition there is a significant spin-orbit coupling in the system, a non zero orbital current density also contributes to the molecular magnetic field density. Such a density is a bona fide, gauge invariant observable, that deserves special attention because it is the genuine observable (and not the spin density) probed in experiments such as polarized neutron diffraction experiments [1].

The purpose of this article is to calculate magnetic field densities and to analyze the respective contributions of the spin density and the orbital current density of $\mathrm{CoCl}_{4}^{2-}$ molecular ions embedded in tricaesium cobalt (II) pentachloride, $\mathrm{Cs}_{3} \mathrm{CoCl}_{5}$, crystals. An extensive bibliography on this system has been provided in previous publication [1,2]. More recent theoretical studies show that it is still the topics of active research [3,4]. In fact, we will limit ourselves to the paramagnetic contribution to the orbital current, the diamagnetic one having been found negligible in our previous studies $[1,2]$, hence we will avoid the delicate problem of gauge invariance, see $[5,6]$ and therein.

Accurate density operators for the $\mathrm{CoCl}_{4}^{2-}$ molecular ions embedded in $\mathrm{Cs}_{3} \mathrm{CoCl}_{5}$ crystals have been derived [1] from polarised neutron diffraction (PND) experiments [7]. Two data sets were collected from PND experiments. The first one, hereafter referred to as the "c-data set", was obtained with an applied magnetic field of $4.6 \mathrm{~T}$ along the c-axis of the cristal, which has the $I 4 / \mathrm{mcm}$ space group symmetry. This axis corresponds to the main symmetry axis (hereafter regarded as the z-axis) of the $\mathrm{CoCl}_{4}^{2-}$ molecular ions, which have $D_{2 d}$ point group symmetry in the cristal. The second data set, called the "a-data set", was obtained with an applied magnetic field of $1.49 \mathrm{~T}$ along the cristal a-axis, which is perpendicular to the c-axis. Correspondingly, two density operators were derived, both of them achieving a goodness of fit, $\chi^{2}$, less then 1 for the difference between calculated and experimental magnetic structure factors. 
In the present study, we use these two density operators to compute magnetic field densities. As far as we are aware, such observables have not been calculated before. So, no benchmark calculation was available. Therefore, for debugging purposes, the calculations were performed with two independent approaches which use different formulas and different computer codes to calculate magnetic field densities in direct space representation. (Note that in previous works, we have calculated magnetic density points in reciprocal space for the sake of comparison with experimental structure factors.) The first one is based on analytical expressions employing the first confluent hypergeometric function [8]. It has been implemented as a Mathematica code [9] and is summarized in Appendix C. The other one is based on Rys-quadrature and will be detailed in the next section and Appendix B. It has been implemented in the computer code TONTO [10], and after checking its results against the first method, it has been used to produce the density maps discussed in Section 3. Our findings will be summarized in conclusion.

\section{Numerical calculation of magnetic field integrals}

Notation: the components $x, y, z$ of three-dimensional vectors, $\vec{v}$, will be denoted as superscript with greek letters, $\left(v^{\alpha}\right)_{\alpha \in\{x, y, z\}}$. The elements of higher-order tensors such as the Krönecker symbol, $\delta_{\alpha \beta}$, (seen as the identity tensor of rank 2), or the Levi-Civita antisymmetric tensor of rank $3, \varepsilon_{\alpha \beta \gamma}$, will be indexed with subscripts. When using the latter tensors, Einstein implicit summation convention will be used for repeated indices.

\subsection{General expressions of the magnetic field integrals}

The magnetic field at point $\vec{r}$ in $3 \mathrm{D}$-space, $\vec{B}[\vec{r}]$, coming from a current density, $\vec{J}[\vec{r}]$, is:

$$
\vec{B}[\vec{r}]=\frac{\mu_{0}}{4 \pi} \vec{\nabla} \times \int \frac{\vec{J}\left[\overrightarrow{r^{\prime}}\right]}{\left\|\overrightarrow{r^{\prime}}-\vec{r}\right\|} d \overrightarrow{r^{\prime}}
$$

The integral can be denoted as $\vec{A}[\vec{r}]$ as it plays the part of a vector potential. The quantity 
$\mu_{0}$ is the permeability of free space, in atomic units $\frac{\mu_{0}}{4 \pi}=\frac{1}{c^{2}}=\alpha^{2}, c$ speed of light, $\alpha \simeq \frac{1}{137}$ fine structure constant.

The electronic current density of a molecule is composed of an "orbital" part and a "spin" part [11]:

$$
\vec{J}[\vec{r}]=\vec{J}_{o}[\vec{r}]+\vec{J}_{s}[\vec{r}]
$$

In terms of an orbital basis set, $\left(\chi_{k}\right)_{k}$, the $\alpha$-component of the spin current density, $\overrightarrow{J_{s}}[\vec{r}]=$ $g_{e} \mu_{e} \vec{\nabla} \times \vec{S}[\vec{r}],\left(g_{e}\right.$, gyromagnetic factor of the electron, $\mu_{e}=-\frac{1}{2}$ au, Bohr magneton $)$, is expressed as,

$$
J_{s}^{\alpha}[\vec{r}]=g_{e} \mu_{e} \varepsilon_{\alpha \beta \gamma} \frac{\partial S^{\gamma}[\vec{r}]}{\partial r^{\beta}}=g_{e} \mu_{e} \varepsilon_{\alpha \beta \gamma} \sum_{k_{1}, \mu ; k_{2}, \nu}\left[\frac{\partial \chi_{k_{1}}^{*}[\vec{r}]}{\partial r^{\beta}} \chi_{k_{2}}[\vec{r}]+\chi_{k_{1}}^{*}[\vec{r}] \frac{\partial \chi_{k_{2}}[\vec{r}]}{\partial r^{\beta}}\right]\left\langle S^{\gamma}\right\rangle_{\mu, \nu} D_{k_{2}, \nu ; k_{1}, \mu}^{(1)}
$$

where $\left\langle S^{\gamma}\right\rangle_{\mu, \nu}$ denotes the matrix element of the spin operator $\gamma$-component, and $D_{k_{2}, \nu ; k_{1}, \mu}^{(1)}$, the one-electron reduced density matrix.

The paramagnetic contribution to the orbital current density is expressed as:

$$
J_{o}^{\alpha}[\vec{r}]=\sum_{k_{1}, k_{2}}\left\langle J_{o}^{\alpha}[\vec{r}]\right\rangle_{k_{1}, k_{2}} D_{k_{2}, k_{1}}^{(1)}
$$

where the current density matrix element is,

$$
\left\langle J_{o}^{\alpha}[\vec{r}]\right\rangle_{k_{1}, k_{2}}=\frac{i}{2}\left[\chi_{k_{1}}^{*}[\vec{r}] \frac{\partial \chi_{k_{2}}[\vec{r}]}{\partial r^{\alpha}}-\frac{\partial \chi_{k_{1}}^{*}[\vec{r}]}{\partial r^{\alpha}} \chi_{k_{2}}[\vec{r}]\right]
$$

and the matrix, $D_{k_{2}, k_{1}}^{(1)}$, is the one-electron reduced density matrix traced over spin variables, 


$$
D_{k_{2}, k_{1}}^{(1)}=\sum_{\mu} D_{k_{2}, \mu ; k_{1}, \mu}^{(1)}
$$

Correspondingly, the magnetic field is comprised of two different origins,

$$
\vec{B}[\vec{r}]=\vec{B}_{o}[\vec{r}]+\vec{B}_{s}[\vec{r}]
$$

with,

$$
\begin{aligned}
& \vec{B}_{o}[\vec{r}]=\frac{1}{c^{2}} \vec{\nabla} \times \int_{\mathbb{R}^{3}} \frac{\overrightarrow{J_{o}}\left[\overrightarrow{r^{\prime}}\right]}{\left\|\overrightarrow{r^{\prime}}-\vec{r}\right\|} d \overrightarrow{r^{\prime}} \\
& \vec{B}_{s}[\vec{r}]=\frac{1}{c^{2}} \vec{\nabla} \times \int_{\mathbb{R}^{3}} \frac{\overrightarrow{J_{o}}\left[\overrightarrow{r^{\prime}}\right]}{\left\|\overrightarrow{r^{\prime}}-\vec{r}\right\|} d \overrightarrow{r^{\prime}} .
\end{aligned}
$$

However, the computation of both contributions boils down to evaluating the same function,

$$
I_{k_{1}, k_{2}}^{\alpha, \beta}[\vec{r}]:=\frac{\partial}{\partial r^{\beta}} \int_{\mathbb{R}^{3}} \chi_{k_{1}}^{*}\left[\overrightarrow{r^{\prime}}\right] \frac{\partial \chi_{k_{2}}\left[\overrightarrow{r^{\prime}}\right]}{\partial r^{\prime \alpha}} \cdot \frac{1}{\left\|\overrightarrow{r^{\prime}}-\vec{r}\right\|} d \overrightarrow{r^{\prime}}
$$

since,

$$
\begin{aligned}
B_{o}^{\alpha}[\vec{r}] & =\frac{\varepsilon_{\alpha \gamma \beta}}{c^{2}} \frac{\partial}{\partial r^{\gamma}} \int_{\mathbb{R}^{3}} \frac{J_{o}^{\beta}\left[\overrightarrow{r^{\prime}}\right]}{\left\|\overrightarrow{r^{\prime}}-\vec{r}\right\|} d \overrightarrow{r^{\prime}} \\
& =\frac{\varepsilon_{\alpha \gamma \beta}}{c^{2}} \sum_{k_{1}, k_{2}} D_{k_{2}, k_{1}}^{(1)} \cdot \frac{\partial}{\partial r^{\gamma}} \int_{\mathbb{R}^{3}} \frac{\left\langle J_{o}^{\beta}\left[\overrightarrow{r^{\prime}}\right]\right\rangle_{k_{1}, k_{2}}}{\left\|\overrightarrow{r^{\prime}}-\vec{r}\right\|} d \overrightarrow{r^{\prime}} \\
& =\frac{\varepsilon_{\alpha \gamma \beta}}{c^{2}} \sum_{k_{1}, k_{2}} D_{k_{2}, k_{1}}^{(1)} \cdot \frac{i}{2} \cdot\left[I_{k_{1}, k_{2}}^{\beta, \gamma}[\vec{r}]-\left(I_{k_{2}, k_{1}}^{\beta, \gamma}[\vec{r}]\right)^{*}\right] \\
& =\frac{\varepsilon_{\alpha \beta \gamma}}{c^{2}} \sum_{k_{1} \neq k_{2}} \operatorname{Im}\left[D_{k_{2}, k_{1}}^{(1)} I_{k_{1}, k_{2}}^{\beta, \gamma}[\vec{r}]\right]
\end{aligned}
$$

(introducing in the last step, the imaginary part $\operatorname{Im}[z]$ of a complex number $z$, and using $\left.\varepsilon_{\alpha \beta \gamma}=-\varepsilon_{\alpha \gamma \beta}\right)$, and, 


$$
\begin{aligned}
B_{s}^{\alpha}[\vec{r}] & =\frac{-g_{e} \mu_{e}}{c^{2}} \varepsilon_{\alpha \beta \gamma} \varepsilon_{\beta \kappa \lambda} \frac{\partial}{\partial r^{\gamma}} \int_{\mathbb{R}^{3}} \frac{\frac{\partial S^{\lambda}\left[\overrightarrow{r^{\prime}}\right]}{\partial r^{\prime \kappa}}}{\left\|\overrightarrow{r^{\prime}}-\vec{r}\right\|} d \overrightarrow{r^{\prime}} \\
& =\frac{-g_{e} \mu_{e}}{c^{2}} \varepsilon_{\alpha \beta \gamma} \varepsilon_{\beta \kappa \lambda} \sum_{k_{1}, \mu ; k_{2}, \nu} D_{k_{2}, \nu ; k_{1}, \mu}^{(1)}\left[I_{k_{1}, k_{2}}^{\kappa, \gamma}[\vec{r}]+\left(I_{k_{2}, k_{1}}^{\kappa, \gamma}[\vec{r}]\right)^{*}\right]\left\langle S^{\lambda}\right\rangle_{\mu, \nu} \\
& =\frac{-2 g_{e} \mu_{e}}{c^{2}}\left(\delta_{\alpha \lambda} \delta_{\gamma \kappa}-\delta_{\alpha \kappa} \delta_{\gamma \lambda}\right) \sum_{k_{1}, \mu ; k_{2}, \nu} \operatorname{Re}\left[D_{k_{2}, \nu ; k_{1}, \mu}^{(1)} I_{k_{1}, k_{2}}^{\kappa, \gamma}[\vec{r}]\left\langle S^{\lambda}\right\rangle_{\mu, \nu}\right] \\
& =\frac{g_{e}}{c^{2}} \sum_{\substack{k_{1}, \mu ; k_{2}, \nu \\
\gamma \neq \alpha}}\left(\operatorname{Re}\left[D_{k_{2}, \nu ; k_{1}, \mu}^{(1)} I_{k_{1}, k_{2}}^{\gamma, \gamma}[\vec{r}]\left\langle S^{\alpha}\right\rangle_{\mu, \nu}\right]-\operatorname{Re}\left[D_{k_{2}, \nu ; k_{1}, \mu}^{(1)} I_{k_{1}, k_{2}}^{\alpha, \gamma}[\vec{r}]\left\langle S^{\gamma}\right\rangle_{\mu, \nu}\right]\right)
\end{aligned}
$$

(introducing the real part, $R e[z]$, of a complex number, $z$, using the identity $\varepsilon_{\alpha \beta \gamma} \varepsilon_{\beta \kappa \lambda}=$ $\delta_{\alpha \lambda} \delta_{\gamma \kappa}-\delta_{\alpha \kappa} \delta_{\gamma \lambda}$ and replacing the Bohr magneton by its value in atomic units). Note that the restriction $\gamma \neq \alpha$ in the summation is not necessary, for the term $\gamma=\alpha$ would be zero anyway.

If the orbital basis functions are real, the $I_{k_{1}, k_{2}}^{\alpha, \beta}[\vec{r}]$ are also real, and can be extracted from the real or imaginary parts in Eqs.(11) and (12),

$$
\begin{aligned}
& B_{o}^{\alpha}[\vec{r}]=\frac{\varepsilon_{\alpha \beta \gamma}}{c^{2}} \sum_{k_{1} \neq k_{2}} \operatorname{Im}\left[D_{k_{2}, k_{1}}^{(1)}\right] I_{k_{1}, k_{2}}^{\beta, \gamma}[\vec{r}], \\
& B_{s}^{\alpha}[\vec{r}]=\frac{g_{e}}{c^{2}} \sum_{\substack{k_{1}, \mu ; k_{2}, \nu \\
\gamma \neq \alpha}}\left(\operatorname{Re}\left[D_{k_{2}, \nu ; k_{1}, \mu}^{(1)}\left\langle S^{\alpha}\right\rangle_{\mu, \nu}\right] I_{k_{1}, k_{2}}^{\gamma, \gamma}[\vec{r}]-\operatorname{Re}\left[D_{k_{2}, \nu ; k_{1}, \mu}^{(1)}\left\langle S^{\gamma}\right\rangle_{\mu, \nu}\right] I_{k_{1}, k_{2}}^{\alpha, \gamma}[\vec{r}]\right) .
\end{aligned}
$$

Recall that the spin matrices can be represented as one half of the Pauli matrices, the above equations give for the $z$-components,

$$
\begin{aligned}
& B_{o}^{z}[\vec{r}]=\frac{1}{c^{2}} \sum_{k_{1} \neq k_{2}} \operatorname{Im}\left[D_{k_{2}, k_{1}}^{(1)}\right]\left(I_{k_{1}, k_{2}}^{x, y}[\vec{r}]-I_{k_{1}, k_{2}}^{y, x}[\vec{r}]\right) \\
& B_{s}^{z}[\vec{r}]=\frac{g_{e}}{2 c^{2}} \sum_{k_{1}, k_{2}} \operatorname{Re}\left[D_{k_{2},+\frac{1}{2} ; k_{1},+\frac{1}{2}}^{(1)}-D_{k_{2},-\frac{1}{2} ; k_{1},-\frac{1}{2}}^{(1)}\right]\left(I_{k_{1}, k_{2}}^{x, x}[\vec{r}]+I_{k_{1}, k_{2}}^{y, y}[\vec{r}]\right) \\
&-\operatorname{Re}\left[D_{k_{2},-\frac{1}{2} ; k_{1},+\frac{1}{2}}^{(1)}+D_{k_{2},+\frac{1}{2} ; k_{1},-\frac{1}{2}}^{(1)}\right] I_{k_{1}, k_{2}}^{z, x}[\vec{r}] \\
&-\operatorname{Im}\left[D_{k_{2},-\frac{1}{2} ; k_{1},+\frac{1}{2}}^{(1)}-D_{k_{2},+\frac{1}{2} ; k_{1},-\frac{1}{2}}^{(1)}\right] I_{k_{1}, k_{2}}^{z, y}[\vec{r}],
\end{aligned}
$$


or for the $x$-components,

$$
\begin{aligned}
& B_{o}^{x}[\vec{r}]=\frac{1}{c^{2}} \sum_{k_{1} \neq k_{2}} \operatorname{Im}\left[D_{k_{2}, k_{1}}^{(1)}\right]\left(I_{k_{1}, k_{2}}^{y, z}[\vec{r}]-I_{k_{1}, k_{2}}^{z, y}[\vec{r}]\right) \\
& B_{s}^{x}[\vec{r}]=\frac{g_{e}}{2 c^{2}} \sum_{k_{1}, k_{2}} \operatorname{Re}\left[D_{k_{2},+\frac{1}{2} ; k_{1},-\frac{1}{2}}^{(1)}+D_{k_{2},-\frac{1}{2} ; k_{1},+\frac{1}{2}}^{(1)}\right]\left(I_{k_{1}, k_{2}}^{z, z}[\vec{r}]+I_{k_{1}, k_{2}}^{y, y}[\vec{r}]\right) \\
&-\operatorname{Im}\left[D_{k_{2},-\frac{1}{2} ; k_{1},+\frac{1}{2}}^{(1)}-D_{k_{2},+\frac{1}{2} ; k_{1},-\frac{1}{2}}^{(1)}\right] I_{k_{1}, k_{2}}^{x, y}[\vec{r}] \\
&-\operatorname{Re}\left[D_{k_{2},+\frac{1}{2} ; k_{1},+\frac{1}{2}}^{(1)}-D_{k_{2},-\frac{1}{2} ; k_{1},-\frac{1}{2}}^{(1)}\right] I_{k_{1}, k_{2}}^{x, z}[\vec{r}] .
\end{aligned}
$$

In the following section we work out some simplifications of the calculation of the $I_{k_{1}, k_{2}}^{\alpha, \beta}[\vec{r}]$ integrals when the orbitals are gaussian functions.

\subsection{Case of Gaussian basis functions}

We now consider primitive Gaussian basis functions, ("Gaussians" in short) of the form,

$$
\chi_{k}[\vec{r}]=\prod_{\alpha \in\{x, y, z\}}\left(r^{\alpha}-R_{k}^{\alpha}\right)^{L_{k}^{\alpha}} \operatorname{Exp}\left[-\zeta_{k}\left(r^{\alpha}-R_{k}^{\alpha}\right)^{2}\right] .
$$

A partial derivative of such a Gaussian function is just a linear combination of two different Gaussians, with the same exponential part but different prefactors,

$$
\frac{\partial \chi_{k}[\vec{r}]}{\partial r^{\alpha}}=\left(\frac{L_{k}^{\alpha}}{r^{\alpha}-R_{k}^{\alpha}}-2 \zeta_{k}\left(r^{\alpha}-R_{k}^{\alpha}\right)\right) \chi_{k}[\vec{r}]
$$

Let us define a function $f$ of two integer triplets $\vec{M}_{i}:=\left(M_{i}^{x}, M_{i}^{y}, M_{i}^{z}\right), i \in\{1,2\}$ and three vectors of $\mathbb{R}^{3}, \vec{R}_{i}:=\left(R_{i}^{x}, R_{i}^{y}, R_{i}^{z}\right), i \in\{1,2\}, \vec{r}:=\left(r^{x}, r^{y}, r^{z}\right)$, by:

$$
f\left[\vec{R}_{1}, \vec{M}_{1}, \vec{R}_{2}, \vec{M}_{2}, \vec{r}\right]:=\prod_{\substack{\alpha \in\{x, y, z\} \\ i \in\{1,2\}}}\left(r^{\alpha}-R_{i}^{\alpha}\right)^{M_{i}^{\alpha}}
$$

(eq.(7) of [8] with a misprint corrected). Denoting by $\vec{e}_{\alpha}$ the unit vector of $\mathbb{R}^{3}$ in direction $\alpha$, the integrals, $I_{k_{1}, k_{2}}^{\alpha, \beta}[\vec{r}]$, can be expressed as, 


$$
\begin{gathered}
I_{k_{1}, k_{2}}^{\alpha, \beta}[\vec{r}]=\frac{\partial}{\partial r^{\beta}} \int_{\mathbb{R}^{3}}\left(L_{k_{2}}^{\alpha} f\left[\vec{R}_{k_{1}}, \vec{L}_{k_{1}}, \vec{R}_{k_{2}}, \vec{L}_{k_{2}}-\vec{e}_{\alpha}, \overrightarrow{r^{\prime}}\right]-2 \zeta_{k_{2}} f\left[\vec{R}_{k_{1}}, \vec{L}_{k_{1}}, \vec{R}_{k_{2}}, \vec{L}_{k_{2}}+\vec{e}_{\alpha}, \overrightarrow{r^{\prime}}\right]\right) \\
\cdot \operatorname{Exp}\left[-\zeta_{k_{1}}\left\|\overrightarrow{r^{\prime}}-\overrightarrow{R_{k_{1}}}\right\|^{2}-\zeta_{k_{2}}\left\|\overrightarrow{r^{\prime}}-\overrightarrow{R_{k_{2}}}\right\|^{2}\right] \cdot \frac{1}{\left\|\overrightarrow{r^{\prime}}-\vec{r}\right\|} d \overrightarrow{r^{\prime}}
\end{gathered}
$$

Using the identity,

$$
\operatorname{Exp}\left[-\zeta_{1}\left\|\vec{r}-\overrightarrow{R_{1}}\right\|^{2}-\zeta_{2}\left\|\vec{r}-\overrightarrow{R_{2}}\right\|^{2}\right]=\operatorname{Exp}\left[\frac{-\zeta_{1} \zeta_{2}}{\zeta_{1}+\zeta_{2}}\left\|\overrightarrow{R_{2}}-\overrightarrow{R_{1}}\right\|^{2}\right] \operatorname{Exp}\left[-\left(\zeta_{1}+\zeta_{2}\right)\left\|\vec{r}-\frac{\zeta_{1} \overrightarrow{R_{1}}+\zeta_{2} \overrightarrow{R_{2}}}{\zeta_{1}+\zeta_{2}}\right\|^{2}\right]
$$

and introducing the function

$$
g^{\beta}\left[\vec{R}_{1}, \vec{M}_{1}, \vec{R}_{2}, \vec{M}_{2}, \zeta_{1}, \zeta_{2}, \vec{r}\right]:=\frac{\partial}{\partial r^{\beta}} \int_{\mathbb{R}^{3}} f\left[\vec{R}_{1}, \vec{M}_{1}, \vec{R}_{2}, \vec{M}_{2}, \vec{r}^{\prime}\right] \times \operatorname{Exp}\left[-\left(\zeta_{1}+\zeta_{2}\right)\left\|\overrightarrow{r^{\prime}}-\frac{\zeta_{1} \vec{R}_{1}+\zeta_{2} \vec{R}_{2}}{\zeta_{1}+\zeta_{2}}\right\|^{2}\right] \frac{d \overrightarrow{r^{\prime}}}{\| \vec{r}-\overrightarrow{r^{\prime} \|}},
$$

we have

$$
\begin{aligned}
I_{k_{1}, k_{2}}^{\alpha, \beta}[\vec{r}]=\operatorname{Exp}\left[\frac{-\zeta_{1} \zeta_{2}}{\zeta_{1}+\zeta_{2}}\left\|\vec{R}_{2}-\vec{R}_{1}\right\|^{2}\right]\left(L_{k_{2}}^{\alpha} g^{\beta}\left[\vec{R}_{1}, \vec{L}_{1}, \vec{R}_{2}, \vec{L}_{2}-\vec{e}_{\alpha}, \zeta_{1}, \zeta_{2}, \vec{r}\right]\right. \\
\left.-2 \zeta_{k_{2}} g^{\beta}\left[\vec{R}_{1}, \vec{L}_{1}, \vec{R}_{2}, \vec{L}_{2}+\vec{e}_{\alpha}, \zeta_{1}, \zeta_{2}, \vec{r}\right]\right) .
\end{aligned}
$$

So, to obtain $I_{k_{1}, k_{2}}^{\alpha, \beta}[\vec{r}]$, it suffices to compute the function, $g^{\beta}\left[\vec{R}_{1}, \vec{M}_{1}, \vec{R}_{2}, \vec{M}_{2}, \zeta_{1}, \zeta_{2}, \vec{r}\right]$, for non-negative integer triplets, $\left(M_{i}^{x}, M_{i}^{y}, M_{i}^{z}\right), i \in\{1,2\}$, as in [8]. In fact, that is just the derivative of a "Coulomb potential" type of integral with Gaussian basis functions, which is easy to evaluate numerically according to known techniques such as Rys quadrature [14]. In Appendix B, we demonstrate that these integrals are actually amenable to an exact calculation with Rys quadrature method, and provide details on how to derive practical formulas using a double quadrature method.

\section{Application to $\mathrm{CoCl}_{4}^{2-}$}

The formulas of the previous section and Appendix B have been implemented in the code TONTO [10] and applied to the density operators optimized in [1] for $\mathrm{CoCl}_{4}^{2-}$ in $\mathrm{Cs}_{3} \mathrm{CoCl}_{5}$ crystals in order to calculate the current and spin density magnetic field at different grid points. The numerical accuracy of the formulas has been checked thoroughly against ana- 
lytical formulas, see ref. [8] and Appendix C. The results are displayed in Figs. 1 and 2 and are analyzed below.

We have concentrated our attention on the components of the magnetic field that have been probed in PND experiments and so have served to optimize our density operators. That is to say, the $z$-component in connection with the c-data set and the $\mathrm{x}$-component in connection with the a-data set. The 2D grids represented in this study correspond to the so-called "small angle plane" and "large angle plane" used in previous studies, that is to say, they are planes containing the cobalt atom and two chlorines forming a $\widehat{C l C o C l}$ angle of either $107.22^{\circ}$ or $110.61^{\circ}$. However, other planes have been used for plots not represented in this article. These plots will be commented on in the text.

Note first that comparing spike height can be misleading. For example, in Fig. 1, the maximum value of the $z$-component of $B_{\text {spin }}$ in the small angle plane that is about $0.000341 \mathrm{au}$, with that of $B_{o r b}$, equal to about 0.000197 , one find a very large ratio, that is $B_{\text {orb }}$ would appear to be more than $50 \%$ of $B_{\text {spin }}$. In fact if one integrates over all 2D-grid points one find a ratio of about $30 \%$. We anticipate that integrating over $3 \mathrm{D}$-space and not just a single plane, would give an orbital versus spin bulk ratio, $\frac{B_{\text {orb }}}{B_{\text {spin }}}$, of the order of $20 \%$, that is similar to the orbital versus spin contribution found for the $g$-value obtained in ESR experiments [13] of 2.42 , this number being also very close to the $g$-value obtained from our optimized density matrix for the c-data set [1], $g=2.46$.

Let us start with the magnetic field z-component of the density operator optimized with the c-data set. In Fig. 1, the upper panels show that the orbital current magnetic field zcomponent is essentially centered on the cobalt atom, slightly stretched along the z-direction that is along the $\mathrm{CoCl}_{4}^{2-}$ tetrahedron elongation. This is easily rationalized from the orbital current density, displayed on Fig. 3 or in [1]. There is no trace of the chlorine contributions to the orbital magnetic field at this scale, whereas the spin density magnetic field z-component displays some small features at their locations at the same scale. In fact, the top of the chlorine spin magnetic field feature is about 15 times higher than that of the orbital one. The orbital current around the chlorine centers appear to be somewhat quenched in the 
$\mathrm{CoCl}_{4}^{2-}$ molecular ion.

In contrast with the orbital magnetic field, the spin magnetic field (4 lower panels of Fig. 1) displays very different features in the small and large angle planes. Although the spin densities look similar in the two planes with four spikes in the interaxis areas (see Fig. 4), only for the large angle plane does the spin magnetic field show 4 spikes. This has to do with the special rôle of the $z$-axis with respect to the z-component of the magnetic field. In fact, the same feature is observed for the $B_{x}$ component in the $(x, y=-z)$-plane in the case of the a-data set (plot not shown in the article). Also, contrary to the orbital contribution, the spin one has significant negative features, in particular in the small angle plane. They are however much smaller than the positive ones, as can be seen by comparing the scales of the middle and lower panels.

Let us now examine the magnetic field $x$-component of the density operator optimized with the a-data set. The scales of the drawings in Fig. 2 are enlarged 10 times with respect to the scales of Fig. 1. The smaller magnetic field obtained for the a-data density operator reflects the smaller magnetization of the system due to a smaller applied magnetic field and a less effective splitting of the degenerate electronic energy levels, see Fig.3 of [1]. The ratio $\frac{B_{\text {orb }}}{B_{s p i n}}$ is remarkably small. It shows that even the orbital current around Co is quenched when the applied magnetic field is perpendicular to the main symmetry axis. As a matter of fact, the current density in the lower panel of Fig. 3 has not the circular distribution exhibited in the upper panel.

The small and large angle planes are fairly equivalent for the $x$-component of the magnetic field. Both ressemble the x-component of the spin density (see Fig. 4) which has four spikes around the cobalt center, the two spikes on the chlorine side being significantly larger then the two others. This can only be related to the bonding interaction between the cobalt central nucleus and the chlorine nuclei. The dissymmetry also exists for the c-data density operator but is less noticeable due to its smaller relative amplitude with respect to spike heights (6 times larger for the c-data spin density).

Finally, we note that there is a negative feature in the spin magnetic field $x$-component 
despite the fact that the spin density $x$-component is negative everywhere (in other words, there is no spin polarization). The same was observed for the c-data spin magnetic field $z$-component, except that, here, the negative feature has a shape similar to the large angle plane lower picture of Fig. 1, but a relative magnitude recalling that of the small angle plane lower picture of Fig. 1. In fact, the 3D shape of the magnetic field is rather complex and difficult to apprehend. For example, in the $x y$-plane (picture not presented here), the c-data spin magnetic field $z$-component displays two positive spikes almost symmetrical to two negative spikes. The orbital magnetic field in the same plane shows a single spike similar to those of Fig. 1 but not distorted, that is with a round base shape.

\section{Conclusion}

We have presented an effective numerical procedure to compute the magnetic field produced at a given point in space by a molecular orbital current or spin density. The formulas have been implemented in the TONTO computer code, a copyleft quantum chemistry freeware [10]. As far as we are aware this observable was not investigated before.

We have studied some selected part of the orbital current and spin density magnetic fields of the $\mathrm{CoCl}_{4}^{2-}$ molecular ions embedded in $\mathrm{Cs}_{3} \mathrm{CoCl}_{5}$ crystals in an external magnetic field. The two contributions are quite different. The orbital one is found more sensitive to the direction of the applied magnetic field than the spin one. Conversely, the spin contribution is found more sensitive to presence of chlorine atoms. Indeed, it can even reveal chemical bonding.

We hope that the present study will trigger interest in this observable, whose calculation has now been made available to the community for any molecular density matrix expressed in terms of a Gaussian atomic basis set. 


\section{Appendix A: Paramagnetic current and spin density of $\mathrm{CoCl}_{4}^{2-}$}

The induced molecular magnetic field densities for $\mathrm{CoCl}_{4}^{2-}$ presented in this article arise from the spin densities and orbital current densities depicted in [1]. Here, they are reproduced from different viewpoints. This is useful to better grasp they shape in 3D-space and makes the article more self-contained.

Only the orbital current around the cobalt nucleus is significant as can be seen from the orbital contribution to the magnetic field. So in Fig. 3, we have only displayed the currents in cubes of length 2 a.u. centered on the Co atom, with a view from the top of applied magnetic field axis. In the case of the c-data set we clearly see that the current follows circles around Co in the xy-plane, hence the round shaped peak of the orbital contribution to the molecular magnetic field (top of Fig. 1). In contrast, for the a-data set there are pairs of small circles superposed to large circles (bottom picture). Hence, the elongated shape of the peak of the orbital magnetic field (top of Fig. 2) which is actually splitted in two at the tip.

Note that, the spin density $y$-component plots were wrong in [1] due to a misprint in a computer code. They are corrected in Fig. 5. Note also, the abuse of notation for the socalled "large angle plane" passing through $\mathrm{Co}, \mathrm{Cl}_{1}$ and $\mathrm{Cl}_{2}$ of Fig.1 in [1], since the axis labelled " $x=-z$ " is in fact approximately " $x=-.95952071 \times z$ " because of the distorsion of the tetrahedron.

(insert Fig. 5 here)

\section{Appendix B: Calculation of Coulomb field integrals using Rys Polynomials}

In this appendix, we explain our implementation of a Rys and Gauss-Hermite double quadra-

ture method, for the calculation of the function, $g^{\beta}\left[\vec{R}_{1}, \vec{M}_{1}, \vec{R}_{2}, \vec{M}_{2}, \zeta_{1}, \zeta_{2}, \vec{r}\right]$, given in Eq. (36). 
Let us first consider its integral part,

$$
h_{\vec{R}_{1}, \vec{M}_{1}, \zeta_{1}, \vec{R}_{2}, \vec{M}_{2}, \zeta_{2}}[\vec{r}]:=\int_{\mathbb{R}^{3}} f\left[\vec{R}_{1}, \vec{M}_{1}, \vec{R}_{2}, \vec{M}_{2}, \overrightarrow{r^{\prime}}\right] \cdot \frac{\operatorname{Exp}\left[-\left(\zeta_{1}+\zeta_{2}\right)\left\|\overrightarrow{r^{\prime}}-\frac{\zeta_{1} \vec{R}_{1}+\zeta_{2} \vec{R}_{2}}{\zeta_{1}+\zeta_{2}}\right\|^{2}\right]}{\left\|\overrightarrow{r^{\prime}}-\vec{r}\right\|} d \overrightarrow{r^{\prime}}
$$

that is just a "Coulomb potential" type of integral with Gaussian basis functions, which is easy to evaluate numerically according to known techniques such as Gaussian quadratures.

More precisely, as a first step, we employ exactly the same algebra used p.13 of Ref. [8] to transform $g^{\beta}\left[\vec{R}_{1}, \vec{M}_{1}, \vec{R}_{2}, \vec{M}_{2}, \zeta_{1}, \zeta_{2}, \vec{r}\right]$. That is to say, we Laplace transform $\frac{1}{\left\|\overrightarrow{r^{\prime}}-\vec{r}\right\|}$,

$$
\frac{1}{\left\|\vec{r}-\overrightarrow{r^{\prime}}\right\|}=\frac{1}{\sqrt{\pi}} \int_{0}^{+\infty} \operatorname{Exp}\left[-v\left\|\vec{r}-\overrightarrow{r^{\prime}}\right\|^{2}\right] \frac{d v}{\sqrt{v}}
$$

make use again of the identity of Eq.(23) and perform three changes of variables to obtain,

$$
\begin{aligned}
& h_{\vec{R}_{1}, \vec{M}_{1}, \zeta_{1}, \vec{R}_{2}, \vec{M}_{2}, \zeta_{2}}[\vec{s}]:=\frac{1}{\sqrt{\pi}\left(\zeta_{1}+\zeta_{2}\right)} \int_{0}^{1} \frac{d \nu}{\sqrt{\nu}} \operatorname{Exp}\left[-\nu\|\vec{s}\|^{2}\right] \int_{\mathbb{R}^{3}} d \overrightarrow{s^{\prime}} \operatorname{Exp}\left[-\left\|\overrightarrow{s^{\prime}}\right\|^{2}\right] . \\
& f\left[\frac{\zeta_{2}\left(\vec{R}_{1}-\vec{R}_{2}\right)}{\zeta_{1}+\zeta_{2}}-\frac{\nu \vec{s}}{\sqrt{\zeta_{1}+\zeta_{2}}}, \vec{M}_{1}, \frac{\zeta_{1}\left(\vec{R}_{2}-\vec{R}_{1}\right)}{\zeta_{1}+\zeta_{2}}-\frac{\nu \vec{s}}{\sqrt{\zeta_{1}+\zeta_{2}}}, \vec{M}_{2}, \sqrt{\frac{1-\nu}{\zeta_{1}+\zeta_{2}}} \overrightarrow{s^{\prime}}\right],
\end{aligned}
$$

where $\vec{s}:=\sqrt{\zeta_{1}+\zeta_{2}} \vec{r}-\frac{\zeta_{1} \vec{R}_{1}+\zeta_{2} \vec{R}_{2}}{\sqrt{\zeta_{1}+\zeta_{2}}}$. (Note that, as in [8], we have assumed that the two integrals could be swapped.) The second integral on $\overrightarrow{s^{\prime}}$ can be calculated by Gauss-Hermite quadrature, whereas the first one suggests the change of variable, $\nu \rightarrow t^{2}$, in order to use the method of Rys polynomials [15-17]:

$$
\begin{gathered}
h_{\vec{R}_{1}, \vec{M}_{1}, \zeta_{1}, \vec{R}_{2}, \vec{M}_{2}, \zeta_{2}}[\vec{s}]:=\frac{2}{\sqrt{\pi}\left(\zeta_{1}+\zeta_{2}\right)} \int_{0}^{1} d t \operatorname{Exp}\left[-t^{2}\|\vec{s}\|^{2}\right] \int_{\mathbb{R}^{3}} d \overrightarrow{s^{\prime}} \operatorname{Exp}\left[-\left\|\overrightarrow{s^{\prime}}\right\|^{2}\right] . \\
f\left[\frac{\zeta_{2}\left(\vec{R}_{1}-\vec{R}_{2}\right)}{\zeta_{1}+\zeta_{2}}-\frac{t^{2} \vec{s}}{\sqrt{\zeta_{1}+\zeta_{2}}}, \vec{M}_{1}, \frac{\zeta_{1}\left(\vec{R}_{2}-\vec{R}_{1}\right)}{\zeta_{1}+\zeta_{2}}-\frac{t^{2} \vec{s}}{\sqrt{\zeta_{1}+\zeta_{2}}}, \vec{M}_{2}, \sqrt{\frac{1-t^{2}}{\zeta_{1}+\zeta_{2}}} \overrightarrow{s^{\prime}}\right],
\end{gathered}
$$

So, as a second step, we use Rys quadrature [16] to calculate the first integral in Eq. (29). Note that we do have a polynomial in $t^{2}$, as required to obtain an exact Rys quadrature, since only even degrees in the $\overrightarrow{s^{\prime}}$-components give non zero contribution after integration on $\overrightarrow{s^{\prime}}$-components. The minimum number of quadrature points or "roots" to have an exact quadrature is the smallest integer larger than half the degree of the polynomial in factor of 
the Gaussian functions, that is to say, in the present case:

$$
n_{\text {roots }}^{\text {Rys }}=\left\lceil\frac{\sum_{\alpha} M_{k_{1}}^{\alpha}+M_{k_{2}}^{\alpha}}{2}\right\rceil
$$

("ceiling" integer value of $\left.\frac{1}{2} \sum_{\alpha} M_{k_{1}}^{\alpha}+M_{k_{2}}^{\alpha}\right)$.

So, we can rewrite exactly Eq. (29) as a discretized Rys sum (switching back to the $\vec{r}$ variable):

$$
\begin{aligned}
& h_{\vec{R}_{1}, \vec{M}_{1}, \zeta_{1}, \vec{R}_{2}, \vec{M}_{2}, \zeta_{2}}[\vec{r}]:=\frac{2}{\sqrt{\pi}\left(\zeta_{1}+\zeta_{2}\right)} \sum_{k} w_{k}^{\mathrm{Rys}}[\vec{r}] \int_{\mathbb{R}^{3}} d \overrightarrow{s^{\prime}} \operatorname{Exp}\left[-\left\|\overrightarrow{s^{\prime}}\right\|^{2}\right] . \\
& f\left[\overrightarrow{R_{1}}-\frac{\zeta_{1} \vec{R}_{1}+\zeta_{2} \vec{R}_{2}+\left(\zeta_{1}+\zeta_{2}\right) u_{k}[\vec{r} \vec{r}}{\left(\zeta_{1}+\zeta_{2}\right)\left(1+u_{k}[\vec{r}]\right)}, \vec{M}_{1}, \overrightarrow{R_{2}}-\frac{\zeta_{1} \vec{R}_{1}+\zeta_{2} \vec{R}_{2}+\left(\zeta_{1}+\zeta_{2}\right) u_{k}[\vec{r}] \vec{r}}{\left(\zeta_{1}+\zeta_{2}\right)\left(1+u_{k}[\vec{r}]\right)}, \vec{M}_{2}, \frac{\overrightarrow{s^{\prime}}}{\sqrt{\left(\zeta_{1}+\zeta_{2}\right)\left(1+u_{k}[\vec{r}]\right)}}\right],
\end{aligned}
$$

where the $u_{k}[\vec{r}]$ 's are related to the roots of the Rys polynomials, the $t_{k}\left[\|\vec{s}\|^{2}\right]^{\prime}$, by, $u_{k}[\vec{r}]=$ $\frac{\left(t_{k}\left[\|\vec{s}\|^{2}\right]\right)^{2}}{1-\left(t_{k}\left[\|\vec{s}\|^{2}\right]\right)^{2}}$, see Ref. [16], and the $w_{k}^{\text {Rys }}[\vec{r}]$ 's are the Rys "weights".

Next, for every $u_{k}[\vec{r}]$ value, we still need to evaluate the integrals,

$$
\begin{aligned}
& N_{\vec{R}_{1}, \vec{M}_{1}, \zeta_{1}, \vec{R}_{2}, \vec{M}_{2}, \zeta_{2}, u_{k}[\vec{r}]}^{\alpha}[\vec{r}]:=\int_{\mathbb{R}} d s^{\prime \alpha} \operatorname{Exp}\left[-s^{\prime \alpha}\right] . \\
& f_{\alpha}\left[R_{1}^{\alpha}-\frac{\zeta_{1} R_{1}^{\alpha}+\zeta_{2} R_{2}^{\alpha}+\left(\zeta_{1}+\zeta_{2}\right) u_{k}[\vec{r}] r^{\alpha}}{\left(\zeta_{1}+\zeta_{2}\right)\left(1+u_{k}[\vec{r}]\right)}, M_{1}^{\alpha}, R_{2}^{\alpha}-\frac{\zeta_{1} R_{1}^{\alpha}+\zeta_{2} R_{2}^{\alpha}+\left(\zeta_{1}+\zeta_{2}\right) u_{k}[\vec{r}] r^{\alpha}}{\left(\zeta_{1}+\zeta_{2}\right)\left(1+u_{k}[\vec{r}]\right)}, M_{2}^{\alpha}, \frac{s^{\prime \alpha}}{\sqrt{\left(\zeta_{1}+\zeta_{2}\right)\left(1+u_{k}[\vec{r}]\right)}}\right],
\end{aligned}
$$

where the function $f_{\alpha}$ denotes the factor of the function $f$ corresponding to $\alpha$-components,

$$
f_{\alpha}\left[R_{1}^{\alpha}, M_{1}^{\alpha}, R_{2}^{\alpha}, M_{2}^{\alpha}, r^{\alpha}\right]:=\prod_{i \in\{1,2\}}\left(r^{\alpha}-R_{i}^{\alpha}\right)^{M_{i}^{\alpha}}
$$

In the last step to obtain the $h_{\vec{R}_{1}, \vec{M}_{1}, \zeta_{1}, \vec{R}_{2}, \vec{M}_{2}, \zeta_{2}}[\vec{r}]$ function, we use exact Gauss-Hermite quadratures for these integrals and replace them by the discrete sums:

$$
\begin{aligned}
& N_{\vec{R}_{1}, \vec{M}_{1}, \zeta_{1}, \vec{R}_{2}, \vec{M}_{2}, \zeta_{2}, u_{k}[\vec{r}]}^{\alpha}[\vec{r}]=\sum_{l} w_{l}^{\mathrm{G}-\mathrm{H}}\left[M_{1}^{\alpha}+M_{2}^{\alpha}\right] \\
& f_{\alpha}\left[R_{1}^{\alpha}-\frac{\zeta_{1} R_{1}^{\alpha}+\zeta_{2} R_{2}^{\alpha}+\left(\zeta_{1}+\zeta_{2}\right) u_{k}[\vec{r}] r^{\alpha}}{\left(\zeta_{1}+\zeta_{2}\right)\left(1+u_{k}[\vec{r}]\right)}, M_{1}^{\alpha}, R_{2}^{\alpha}-\frac{\zeta_{1} R_{1}^{\alpha}+\zeta_{2} R_{2}^{\alpha}+\left(\zeta_{1}+\zeta_{2}\right) u_{k}[\vec{r}] r^{\alpha}}{\left(\zeta_{1}+\zeta_{2}\right)\left(1+u_{k}[\vec{r}]\right)}, M_{2}^{\alpha}, \frac{s_{l}^{\prime \alpha}\left[M_{1}^{\alpha}+M_{2}^{\alpha}\right]}{\sqrt{\left(\zeta_{1}+\zeta_{2}\right)\left(1+u_{k}[\vec{r}]\right)}}\right],
\end{aligned}
$$


where the Gauss-Hermite roots, $s_{l}^{\prime \alpha}\left[M_{1}^{\alpha}+M_{2}^{\alpha}\right]$, and weights, $w_{l}^{\mathrm{G}-\mathrm{H}}\left[M_{1}^{\alpha}+M_{2}^{\alpha}\right]$, depends on the minimal number of quadrature points required to have an exact result, that is to say, upon the degree of the polynomial in $s^{\prime \alpha}$, hence their dependence upon $M_{1}^{\alpha}+M_{2}^{\alpha}$. In fact, the number of points required is

$$
n_{\text {roots }}^{\mathrm{G}-\mathrm{H}}=\left\lceil\frac{M_{k_{1}}^{\alpha}+M_{k_{2}}^{\alpha}}{2}\right\rceil
$$

Routines to calculate these weights and roots are widely available, see for example [18].

Let us return to the calculation of the function $g^{\beta}$,

$$
g^{\beta}\left[\vec{R}_{1}, \vec{M}_{1}, \vec{R}_{2}, \vec{M}_{2}, \zeta_{1}, \zeta_{2}, \vec{r}\right]=\frac{\partial h_{\vec{R}_{1}, \vec{M}_{1}, \zeta_{1}, \vec{R}_{2}, \vec{M}_{2}, \zeta_{2}}[\vec{r}]}{\partial r^{\beta}}
$$

It can be regarded as a "Coulomb field" integral type, since it is a derivative of a "Coulomb potential" integral. Looking back at Eq.(26), we see that only $\frac{1}{\left\|\overrightarrow{r^{\prime}}-\vec{r}\right\|}$ needs to be differentiated, and assuming that derivation permutes with the Laplace transform, Eq. (27), we get:

$$
\frac{\partial\left\|\vec{r}-\vec{r}^{\prime}\right\|^{-1}}{\partial r^{\beta}}=\frac{1}{\sqrt{\pi}} \int_{0}^{+\infty}-2 v\left(r^{\beta}-r^{\prime \beta}\right) \operatorname{Exp}\left[-v\left\|\vec{r}-\overrightarrow{r^{\prime}}\right\|^{2}\right] d v .
$$

So, there is only an additional factor of $-2 v\left(r^{\beta}-r^{\prime \beta}\right)$ to take into account with respect to the calculation of $h_{\vec{R}_{1}, \vec{M}_{1}, \zeta_{1}, \vec{R}_{2}, \vec{M}_{2}, \zeta_{2}}[\vec{r}]$. After the successive changes of variables, this factor becomes $-2\left(\zeta_{1}+\zeta_{2}\right) t^{2}\left(r^{\beta}-\frac{\zeta_{1} R_{1}^{\beta}+\zeta_{2} R_{2}^{\beta}}{\zeta_{1}+\zeta_{2}}-\frac{s^{\prime \beta}}{\sqrt{\left(\zeta_{1}+\zeta_{2}\right)\left(1-t^{2}\right)}}\right)$. This seems to introduce a factor $\frac{1}{\sqrt{1-t^{2}}}$, however, as we have already stated, only even powers of $s^{\prime \beta}$ give non zero contributions to the integral over $s^{\prime \beta}$, and all other factors $s^{\prime \beta}$ in $f_{\beta}$ comes with a factor $\sqrt{1-t^{2}}$, see Eq. (29). So, again the function to integrate over $t$ will be a polynomial in $t^{2}$ amenable to exact Rys quadrature. The only thing to pay attention to is that its degree is increased by one with respect to the case of Coulomb potential integrals, hence the required number of roots will have to be increased accordingly. (Note however, that the above argument would not to apply beyond Coulomb potential integral first derivatives.)

For each Rys quadrature point, the extra factor can be expressed as $-2\left(\zeta_{1}+\zeta_{2}\right) u_{k}[\vec{r}]\left(r^{\beta}-\frac{s_{l}^{\prime \beta}\left[M_{1}^{\beta}+M_{2}^{\beta}\right]}{\sqrt{\left(\zeta_{1}+\zeta_{2}\right)\left(1+u_{k}[\vec{r}]\right)}}-\frac{\zeta_{1} R_{1}^{\beta}+\zeta_{2} R_{2}^{\beta}+\left(\zeta_{1}+\zeta_{2}\right) u_{k}[\vec{r}] r^{\beta}}{\left(\zeta_{1}+\zeta_{2}\right)\left(1+u_{k}[\vec{r}]\right)}\right)$. This factor is to be inserted in the sum of Eq. (34) for $\alpha=\beta$, giving the new function, 


$$
\begin{aligned}
& F_{\vec{R}_{1}, \vec{M}_{1}, \zeta_{1}, \vec{R}_{2}, \vec{M}_{2}, \zeta_{2}, u_{k}[\vec{r}]}^{\beta}[\vec{r}]:=-2\left(\zeta_{1}+\zeta_{2}\right) u_{k}[\vec{r}] \\
& f_{l} w_{l}^{G-\mathrm{H}}\left[M_{1}^{\beta}+M_{2}^{\beta}\right]\left(r^{\beta}-\frac{s_{l}^{\prime \beta}\left[M_{1}^{\beta}+M_{2}^{\beta}\right]}{\sqrt{\left(\zeta_{1}+\zeta_{2}\right)\left(1+u_{k}[\vec{r}]\right)}}-\frac{\zeta_{1} R_{1}^{\beta}+\zeta_{2} R_{2}^{\beta}+\left(\zeta_{1}+\zeta_{2}\right) u_{k}[\vec{r}] r^{\beta}}{\left(\zeta_{1}+\zeta_{2}\right)\left(1+u_{k}[\vec{r}]\right)}\right) \\
& f_{\beta}\left[R_{1}^{\beta}-\frac{\zeta_{1} R_{1}^{\beta}+\zeta_{2} R_{2}^{\beta}+\left(\zeta_{1}+\zeta_{2}\right) u_{k}[\vec{r}] r^{\beta}}{\left(\zeta_{1}+\zeta_{2}\right)\left(1+u_{k}[\vec{r}]\right)}, M_{1}^{\beta}, R_{2}^{\beta}-\frac{\zeta_{1} R_{1}^{\beta}+\zeta_{2} R_{2}^{\beta}+\left(\zeta_{1}+\zeta_{2}\right) u_{k}[\vec{r}] r^{\beta}}{\left(\zeta_{1}+\zeta_{2}\right)\left(1+u_{k}[\vec{r}]\right)}, M_{2}^{\beta}, \frac{s_{l}^{\beta}\left[M_{1}^{\beta}+M_{2}^{\beta}\right]}{\sqrt{\left(\zeta_{1}+\zeta_{2}\right)\left(1+u_{k}[\vec{r}]\right)}}\right],
\end{aligned}
$$

the other two sums, for $\alpha \neq \beta$, remaining unchanged. So, the function $g^{\beta}\left[\vec{R}_{1}, \vec{M}_{1}, \vec{R}_{2}, \vec{M}_{2}, \zeta_{1}, \zeta_{2}, \vec{r}\right]$ is obtained by multiplying these three Gauss-Hermite sums for every Rys quadrature point, and then, by computing the Rys sum,

$$
\begin{aligned}
& g^{\beta}\left[\vec{R}_{1}, \vec{M}_{1}, \vec{R}_{2}, \vec{M}_{2}, \zeta_{1}, \zeta_{2}, \vec{r}\right]= \\
& \quad \frac{2}{\sqrt{\pi}\left(\zeta_{1}+\zeta_{2}\right)} \sum_{k} w_{k}^{\mathrm{Rys}}[\vec{r}] F_{\vec{R}_{1}, \vec{M}_{1}, \zeta_{1}, \vec{R}_{2}, \vec{M}_{2}, \zeta_{2}, u_{k}[\vec{r}]}^{\beta}[\vec{r}] \prod_{\alpha \neq \beta} N_{\vec{R}_{1}, \vec{M}_{1}, \zeta_{1}, \vec{R}_{2}, \vec{M}_{2}, \zeta_{2}, u_{k}[\vec{r}]}^{\alpha}[\vec{r}] .
\end{aligned}
$$

\section{Appendix C: Analytical calculation of magnetic field integrals}

We provide here the analytical formulas against which our numerical calculations of magnetic field density have been checked. They correct some misprints and slight errors of [8] already noted in [19].

The orbital paramagnetic current, $\vec{J}_{o}[\vec{r}]$ produced by the complex combination of primitive Gaussian atomic orbitals

$$
b_{1} \prod_{\alpha \in\{x, y, z\}}\left(r^{\alpha}-R_{1}^{\alpha}\right)^{L_{1}^{\alpha}} \operatorname{Exp}\left[-\zeta_{1}\left(r^{\alpha}-R_{1}^{\alpha}\right)^{2}\right]+i b_{2} \prod_{\alpha \in\{x, y, z\}}\left(r^{\alpha}-R_{2}^{\alpha}\right)^{L_{2}^{\alpha}} \operatorname{Exp}\left[-\zeta_{2}\left(r^{\alpha}-R_{2}^{\alpha}\right)^{2}\right],
$$

( $b_{1}$ and $b_{2}$ are real coefficients) give rise to a molecular magnetic field, whose $\gamma$-component is,

$$
\begin{gathered}
B_{o}^{\gamma}[\vec{r}]=\frac{-\mu_{0}}{4 \pi} \varepsilon_{\alpha \beta \gamma} \frac{\partial}{\partial r^{\beta}} \int_{\mathbb{R}^{3}} \frac{d \vec{r}^{\prime}}{\left\|\vec{r}-\vec{r}^{\prime}\right\|} J_{o}^{\alpha}\left[\overrightarrow{r^{\prime}}\right] \\
=\frac{-\mu_{0}}{4 \pi} \varepsilon_{\alpha \beta \gamma} b_{1} b_{2} \operatorname{Exp}\left[-\frac{\zeta_{1} \zeta_{2}}{\zeta_{1}+\zeta_{2}}\left\|\vec{R}_{1}-\vec{R}_{2}\right\|^{2}\right]\left(L_{1}^{\alpha} g^{\beta}\left[\vec{R}_{1}, \vec{L}_{1}-\vec{e}_{\alpha}, \vec{R}_{2}, \vec{L}_{2}, \zeta_{1}, \zeta_{2}, \vec{r}\right]-L_{2}^{\alpha} g^{\beta}\left[\vec{R}_{1}, \vec{L}_{1}, \vec{R}_{2}, \vec{L}_{2}-\vec{e}_{\alpha}, \zeta_{1}, \zeta_{2}, \vec{r}\right]\right. \\
\left.-2 \zeta_{1} g^{\beta}\left[\vec{R}_{1}, \vec{L}_{1}+\vec{e}_{\alpha}, \vec{R}_{2}, \vec{L}_{2}, \zeta_{1}, \zeta_{2}, \vec{r}\right]+2 \zeta_{2} g^{\beta}\left[\vec{R}_{1}, \vec{L}_{1}, \vec{R}_{2}, \vec{L}_{2}+\vec{e}_{\alpha}, \zeta_{1}, \zeta_{2}, \vec{r}\right]\right)
\end{gathered}
$$

(correcting a sign error in Eq.(9) of [8], Eq.(8) being correct). 
After setting $\vec{S}_{i}=\frac{\overrightarrow{R_{i}}}{\sqrt{\zeta_{1}+\zeta_{2}}}, i \in\{1,2\}, \vec{s}=\sqrt{\zeta_{1}+\zeta_{2}} \vec{r}-\zeta_{1} \overrightarrow{S_{1}}-\zeta_{2} \overrightarrow{S_{2}}$ and some manipulations, the $g^{\beta}$ functions can be re-expressed as,

$$
\begin{gathered}
g^{\beta}\left[\vec{S}_{1}, \vec{M}_{1}, \vec{S}_{2}, \vec{M}_{2}, \zeta_{1}, \zeta_{2}, \vec{s}\right]=\left(\pi\left(\zeta_{1}+\zeta_{2}\right)^{1+\Sigma_{i, \alpha} M_{i}^{\alpha}}\right)^{-\frac{1}{2}} \sum_{\substack{l_{i}+m_{i}+\vec{n}_{i}=\vec{M}_{i} \\
0 \leq l_{i}^{\alpha}, m_{i}^{\alpha}, n_{i}^{\alpha}}}\left(+\zeta_{2}\right)^{\Sigma_{\alpha} n_{1}^{\alpha}}\left(-\zeta_{1}\right)^{\Sigma_{\alpha} n_{2}^{\alpha}} \Gamma\left[1+\sum_{i, \alpha} \frac{l_{i}^{\alpha}}{2}\right] \\
\left(\prod_{\alpha} \delta_{l_{1}^{\alpha}+l_{2}^{\alpha}, 0}^{[2]} \Gamma\left[\frac{l_{1}^{\alpha}+l_{2}^{\alpha}+1}{2}\right]\left(S_{2}^{\alpha}-S_{1}^{\alpha}\right)^{n_{1}^{\alpha}+n_{2}^{\alpha}} \prod_{i} \frac{M_{i}^{\alpha !}}{l_{i}^{\alpha} ! m_{i}^{\alpha} ! n_{i}^{\alpha !} !}\left(s^{\alpha}\right)^{m_{i}^{\alpha}}\right)\left(\frac{m_{1}^{\beta}+m_{2}^{\beta}}{s^{\beta}} \frac{\Gamma\left[\frac{1}{2}+\sum_{i, \alpha} m_{i}^{\alpha}\right]}{\Gamma\left[\frac{3}{2}+\sum_{i, \alpha} m_{i}^{\alpha}+\frac{l_{i}^{\alpha}}{2}\right]}\right. \\
\left.\Phi\left[\frac{1}{2}+\sum_{i, \alpha} m_{i}^{\alpha}, \frac{3}{2}+\sum_{i, \alpha} m_{i}^{\alpha}+\frac{l_{i}^{\alpha}}{2} ;-\|\vec{s}\|^{2}\right]-2 s^{\beta} \frac{\Gamma\left[\frac{3}{2}+\sum_{i, \alpha} m_{i}^{\alpha}\right]}{\Gamma\left[\frac{5}{2}+\sum_{i, \alpha} m_{i}^{\alpha}+\frac{l_{i}^{\alpha}}{2}\right]} \Phi\left[\frac{3}{2}+\sum_{i, \alpha} m_{i}^{\alpha}, \frac{5}{2}+\sum_{i, \alpha} m_{i}^{\alpha}+\frac{l_{i}^{\alpha}}{2} ;-\|\vec{s}\|^{2}\right]\right)
\end{gathered}
$$

where $\delta_{i, j}^{[2]}$ is the Kronecker symbol modulo $2, \Gamma[z]$ is the gamma function, and $\Phi[a, c ; z]$ is the confluent hypergeometric function also denoted by ${ }_{1} F_{1}$,

$$
\Phi[a, c ; z]=\frac{\Gamma[c]}{\Gamma[a] \Gamma[c-a]} \int_{0}^{1} d \nu \nu^{a-1}(1-\nu)^{c-a-1} \operatorname{Exp}[\nu z]
$$

which fulfills the property,

$$
\frac{d \Phi[a, c ; z]}{z}=\frac{a}{c} \Phi[a+1, c+1 ; z]
$$

(the last two equations correct Eqs.(15) and (16) of [8]).

The expression Eq. (41) can be used to compute the expectation value of the magnetic field density from an orbital current, $\left\langle B_{o}^{\gamma}[r]\right\rangle_{D}$ corresponding to a given density operator $D$. If we rewrite the left-hand side of Eq. (41) as $\left\langle B_{o}^{\gamma}[r]\right\rangle_{b_{1}, b_{2}, \vec{R}_{1}, \vec{R}_{2}, \vec{L}_{1}, \vec{L}_{2}, \zeta_{1}, \zeta_{2}}$ and denote by $D^{(1)}$ the one-electron reduced density operator of $D$ represented in some contracted Gaussian basis set $\left(\phi_{k}:=\sum_{j} b_{j, k} \prod_{\alpha \in\{x, y, z\}}\left(r^{\alpha}-R_{k}^{\alpha}\right)^{L_{k}^{\alpha}} \operatorname{Exp}\left[-\zeta_{j, k}\left(r^{\alpha}-R_{1}^{\alpha}\right)^{2}\right]\right)_{k}$, we have,

$$
\left\langle B_{o}^{\gamma}[r]\right\rangle_{D}=\sum_{\substack{k_{1}<k_{2} \\ \mu= \pm \frac{1}{2}}} \operatorname{Im}\left[D_{k_{2}, \mu ; k_{1}, \mu}^{(1)}\right] \sum_{j_{1}, j_{2}}\left\langle B_{o}^{\gamma}[r]\right\rangle_{b_{j_{1}, k_{1}}, b_{j_{1}, k_{2}}, \vec{R}_{k_{1}}, \vec{R}_{k_{2}}, \vec{L}_{k_{1}}, \vec{L}_{k_{2}}, \zeta_{j_{1}, k_{1}}, \zeta_{j_{2}, k_{2}}}
$$


The matrix element between two real spin-orbitals, $\phi_{1, \mu}$ and $\phi_{2, \nu},\left(\mu, \nu= \pm \frac{1}{2}\right)$, of the current density associated to a spin density,

$$
\left\langle\vec{J}_{s}[r]\right\rangle_{\phi_{1, \mu}, \phi_{2, \nu}}=g_{e} \mu_{e} \nabla \times \vec{S}_{\mu, \nu}\left(\phi_{1} \phi_{2}\right)[\vec{r}]
$$

involves the same quantities, $\phi_{i} \frac{\partial}{\partial r^{\beta}} \phi_{j}$, as the orbital current (see Eq.(3) of [8]), but combined with a plus sign instead of a minus,

$$
\left\langle J_{s}^{\gamma}[r]\right\rangle_{\phi_{1, \mu}, \phi_{2, \nu}}=+\frac{g}{2} \varepsilon_{\alpha \beta \gamma} S_{\mu, \nu}^{\alpha}\left(\phi_{1} \frac{\partial}{\partial r^{\beta}} \phi_{2}+\phi_{2} \frac{\partial}{\partial r^{\beta}} \phi_{1}\right)[\vec{r}],
$$

(which corrects a sign error in Eq.(19) of [8]). Note that a factor $-\frac{1}{2}$ arises from the Bohr magneton in atomic units, the minus sign being eliminated by swapping two indices in the Levi-Civita symbol.

As a consequence, the spin magnetic field density, whose matrix elements, for uncontracted Gaussian functions $\phi_{1}$ and $\phi_{2}$, are expressed in terms of the same $g$-functions,

$$
\begin{gathered}
\left\langle B_{s}^{\omega}[\vec{r}]\right\rangle_{b_{1}, b_{2}, \vec{R}_{1}, \vec{R}_{2}, \vec{L}_{1}, \vec{L}_{2}, \zeta_{1}, \zeta_{2}, \mu, \nu}=\frac{-\mu_{0}}{4 \pi} \varepsilon_{\gamma \delta \omega} \frac{\partial}{\partial r^{\delta}} \int_{\mathbb{R}^{3}} \frac{d \overrightarrow{r^{\prime}}}{\left\|\vec{r}-\vec{r}^{\prime}\right\|} J_{s}^{\gamma}\left[\vec{r}^{\prime}\right] \\
=\frac{-g \mu_{0}}{8 \pi} b_{1} b_{2} \varepsilon_{\alpha \beta \gamma} \varepsilon_{\gamma \delta \omega} S_{\mu, \nu}^{\alpha} \operatorname{Exp}\left[-\frac{\zeta_{1} \zeta_{2}}{\zeta_{1}+\zeta_{2}}\left\|\vec{R}_{1}-\vec{R}_{2}\right\|^{2}\right]\left(L_{1}^{\beta} g^{\delta}\left[\vec{R}_{1}, \vec{L}_{1}-\vec{e}_{\beta}, \vec{R}_{2}, \vec{L}_{2}, \zeta_{1}, \zeta_{2}, \vec{r}\right]+L_{2}^{\beta} g^{\delta}\left[\vec{R}_{1}, \vec{L}_{1}, \vec{R}_{2}, \vec{L}_{2}-\vec{e}_{\beta}, \zeta_{1}, \zeta_{2}, \vec{r}\right]\right. \\
\left.-2 \zeta_{1} g^{\delta}\left[\vec{R}_{1}, \vec{L}_{1}+\vec{e}_{\beta}, \vec{R}_{2}, \vec{L}_{2}, \zeta_{1}, \zeta_{2}, \vec{r}\right]-2 \zeta_{2} g^{\delta}\left[\vec{R}_{1}, \vec{L}_{1}, \vec{R}_{2}, \vec{L}_{2}+\vec{e}_{\beta}, \zeta_{1}, \zeta_{2}, \vec{r}\right]\right)
\end{gathered}
$$

is, in some sense, dual to the orbital magnetic field density, as it "probes" the real part of spin-diagonal density matrix elements instead of their imaginary part,

$$
\begin{aligned}
& \left\langle B_{s}^{\gamma}[r]\right\rangle_{D}=\sum_{\substack{k_{1}, k_{2} \\
\mu, \nu= \pm \frac{1}{2}}} D_{k_{2}, \nu ; k_{1}, \mu}^{(1)} \sum_{j_{1}, j_{2}}\left\langle B_{s}^{\gamma}[r]\right\rangle_{b_{j_{1}, k_{1}}, b_{j_{1}, k_{2}}, \vec{R}_{k_{1}}, \vec{R}_{k_{2}}, \vec{L}_{k_{1}}, \vec{L}_{k_{2}}, \zeta_{j_{1}, k_{1}}, \zeta_{j_{2}, k_{2}, \mu, \nu}} \\
= & \sum_{\substack{k_{1} \leq k_{2} \\
\mu, \nu= \pm \frac{1}{2}}} \frac{2}{1+\delta_{k_{1}, k_{2}}} R e\left[D_{k_{2}, \nu ; k_{1}, \mu}^{(1)}\right] \sum_{j_{1}, j_{2}}\left\langle B_{s}^{\gamma}[r]\right\rangle_{b_{j_{1}, k_{1}}, b_{j_{1}, k_{2}}, \vec{R}_{k_{1}}, \vec{R}_{k_{2}}, \vec{L}_{k_{1}}, \vec{L}_{k_{2}}, \zeta_{j_{1}, k_{1}}, \zeta_{j_{2}, k_{2}, \mu, \nu}}
\end{aligned}
$$

Of course, in addition, it also involve the spin-non diagonal matrix elements because of the presence of $S^{x}$ and $S^{y}$ in Eq.(48). So, it is important to study both the orbital and spin magnetic densities, which support complementary informations about a molecular electronic state.

Remark: In our notation, $\left\langle B_{o}^{\gamma}[r]\right\rangle_{b_{1}, b_{2}, \vec{R}_{1}, \vec{R}_{2}, \vec{L}_{1}, \vec{L}_{2}, \zeta_{1}, \zeta_{2}}$ denotes the expectation value over the complex Gaussian orbital of Eq. (40), whereas $\left\langle B_{s}^{\gamma}[r]\right\rangle_{b_{1}, b_{2}, \vec{R}_{1}, \vec{R}_{2}, \vec{L}_{1}, \vec{L}_{2}, \zeta_{1}, \zeta_{2}, \mu, \nu}$ denotes the matrix 
element between two real Gaussian spin-orbitals. 


\section{Figures}
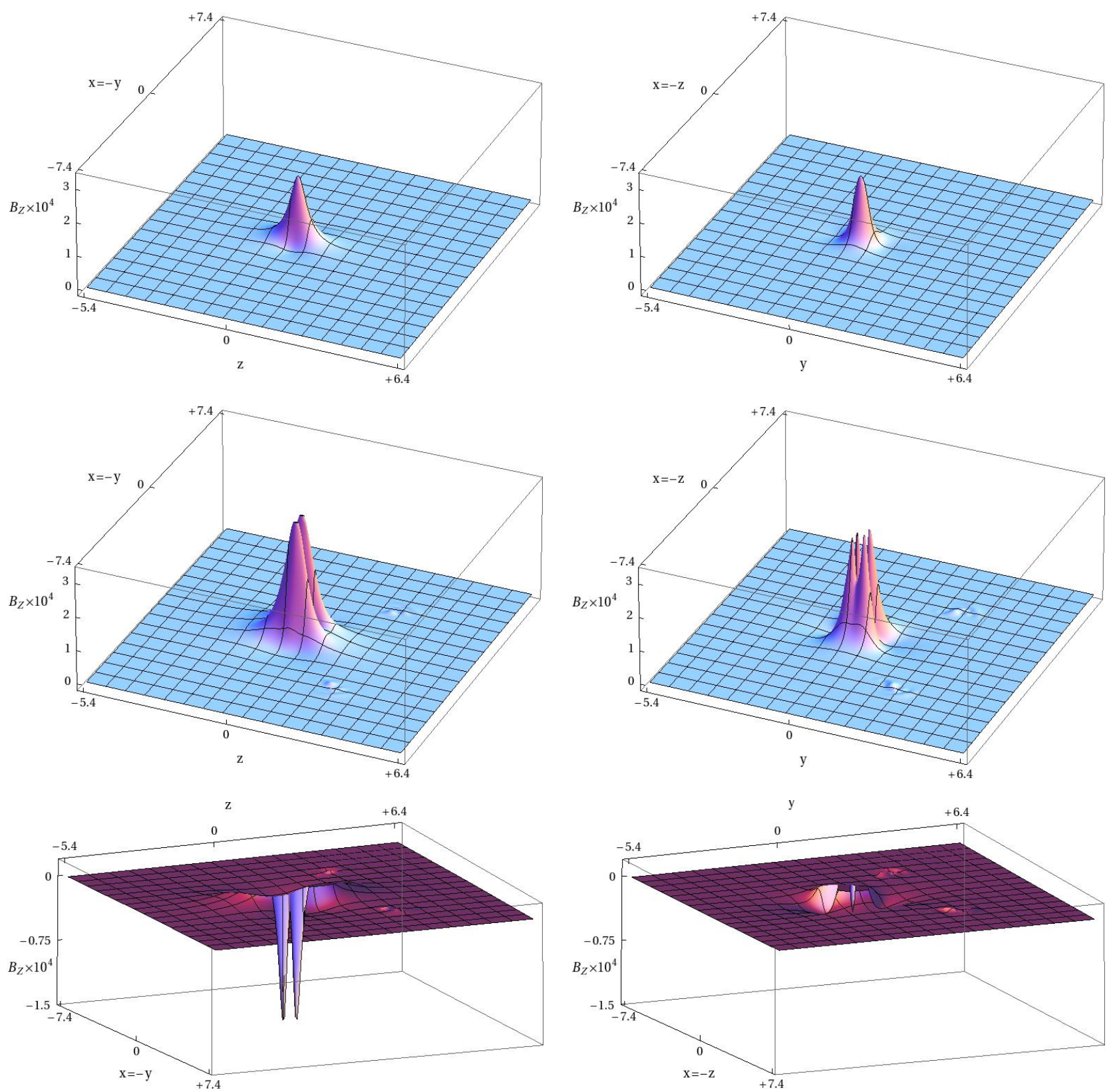

Fig. 1. Component aligned with neutron polarization of the magnetic field density in the case of an applied magnetic field along the $\mathrm{Cs}_{3} \mathrm{CoCl}_{5}$ crystal c-axis (all quantities are in atomic units). Top two figures show the contribution due to the paramagnetic current of $\mathrm{CoCl}_{4}^{2-}$, the four figures below them, that due to its spin density. The right-hand side figures correspond to the $\mathrm{ClCoCl}$ large angle plane defined by $\mathrm{Cl}_{1}$, Co and $\mathrm{Cl}_{2}$ in Fig.1 of [1], the left-hand side figures to the small angle plane defined by $\mathrm{Cl}_{2}$, Co and $\mathrm{Cl}_{3}$. 

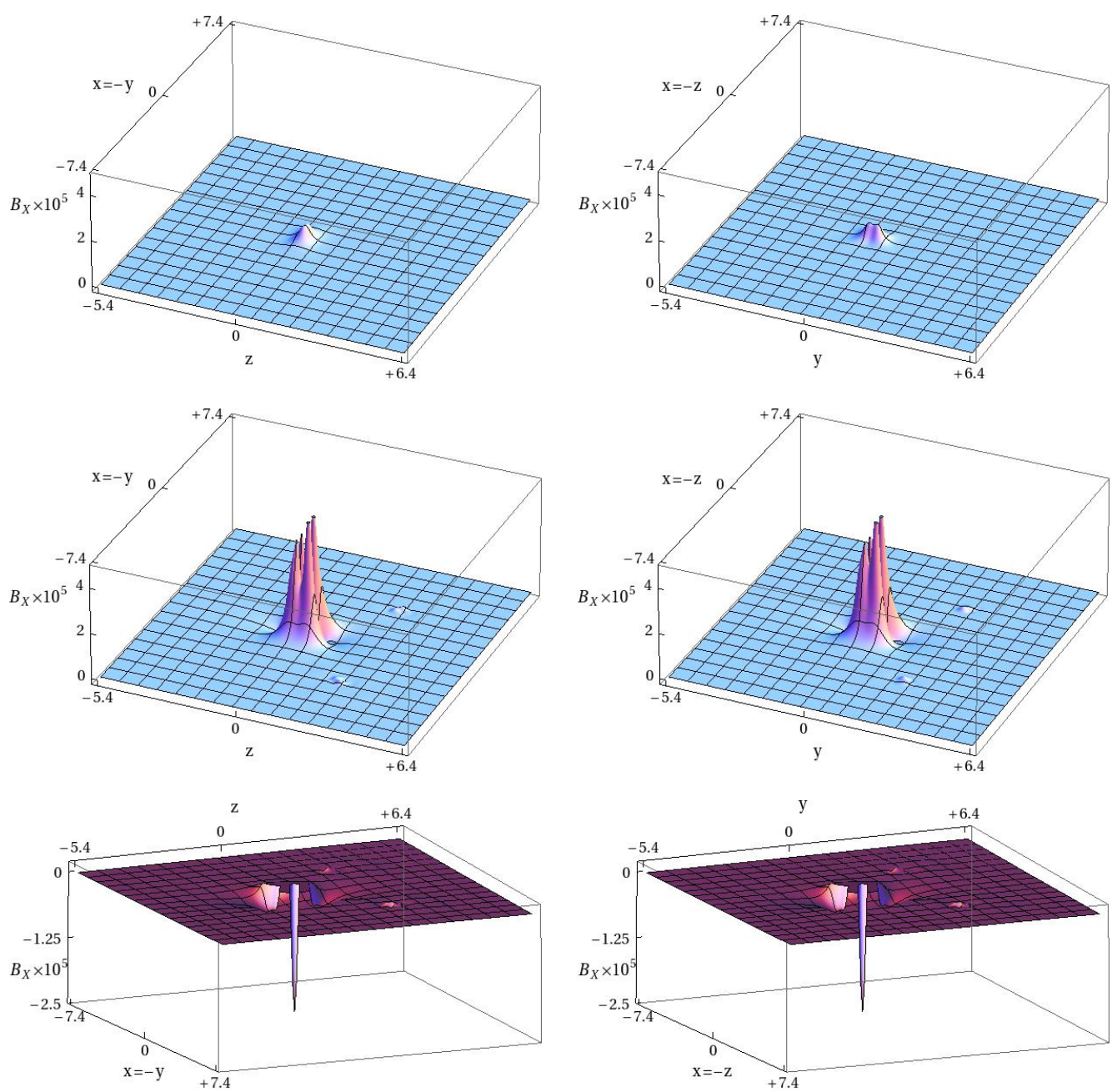

Fig. 2. Component aligned with neutron polarization of the magnetic field density in the case of an applied magnetic field along the $\mathrm{Cs}_{3} \mathrm{CoCl}_{5}$ crystal $a$-axis (all quantities are in atomic units). Top two figures show the contribution due to the paramagnetic current of $\mathrm{CoCl}_{4}^{2-}$, the four figures below them, that due to its spin density. The right-hand side figures correspond to the $\mathrm{ClCoCl}$ large angle plane defined by $\mathrm{Cl}_{1}, \mathrm{Co}$ and $\mathrm{Cl}_{2}$ in Fig.1 of [1], the left-hand side figures to the small angle plane defined by $\mathrm{Cl}_{2}$, $\mathrm{Co}$ and $\mathrm{Cl}_{3}$. 


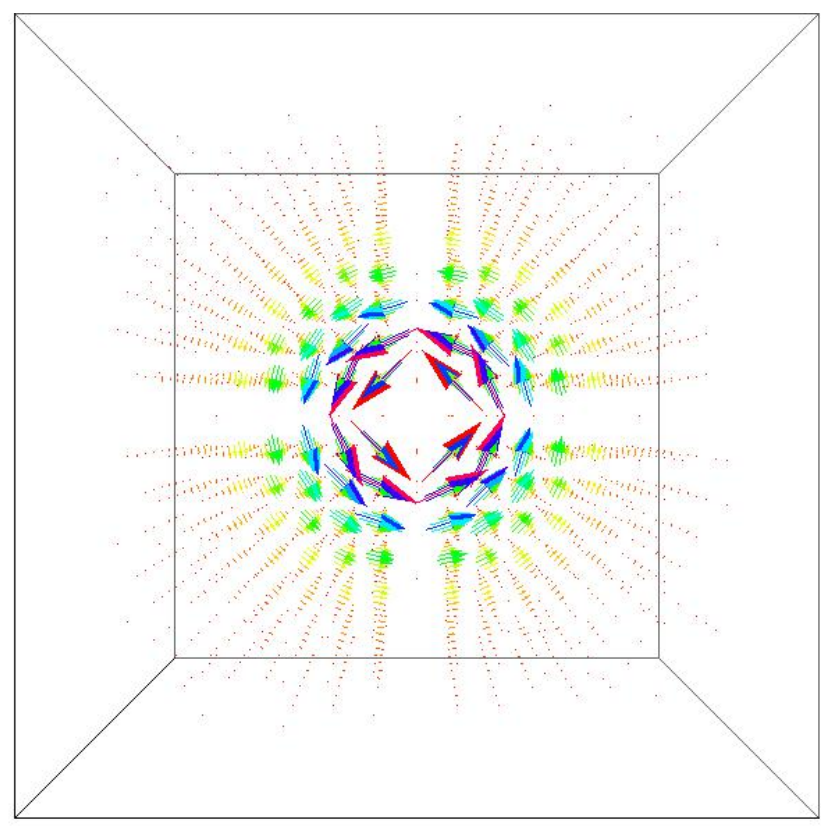

(c-data optimized density operator)

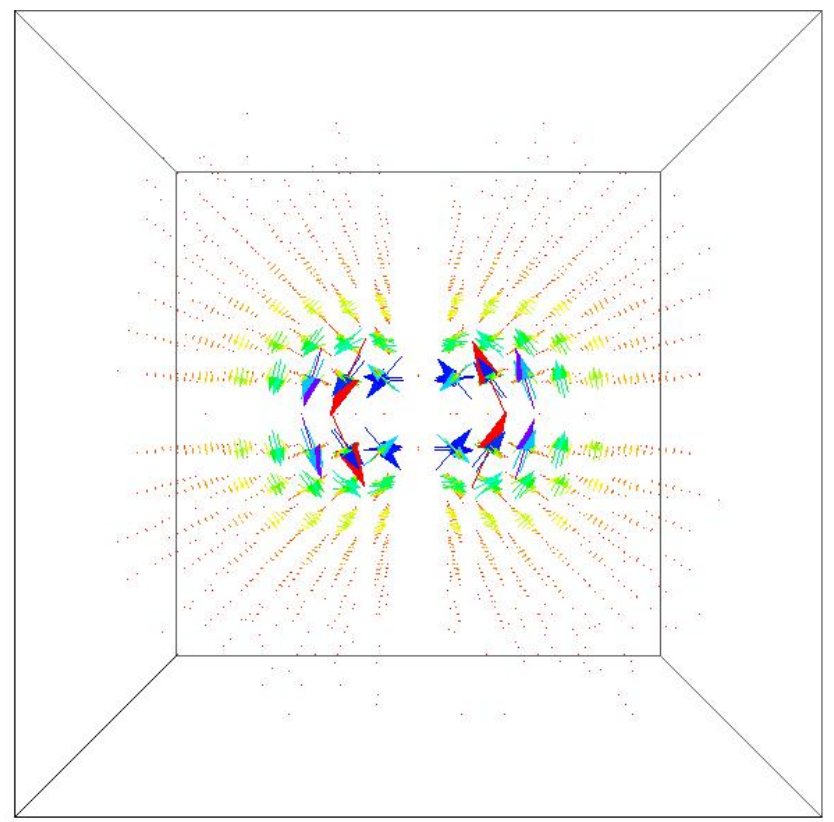

(a-data optimized density operator)

Fig. 3. Paramagnetic current around the Co nucleus in atomic units. The cubes are centered on the cobalt nucleus, their edges are 2 a.u. long. The currents are seen from above the applied magnetic field axis. 


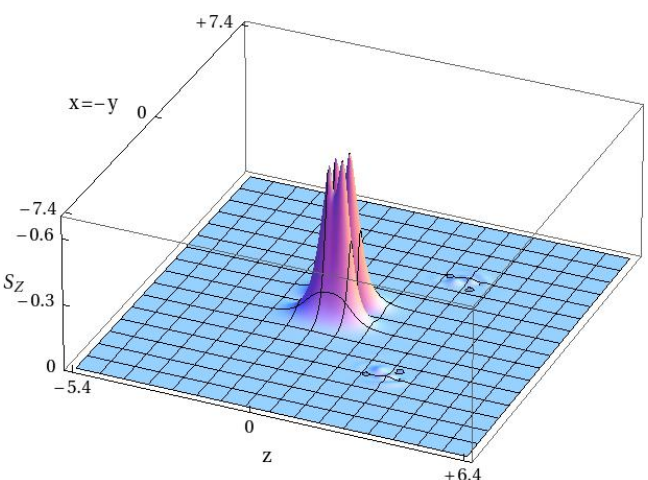

(c-data, $\mathrm{ClCoCl}$ small angle plane)

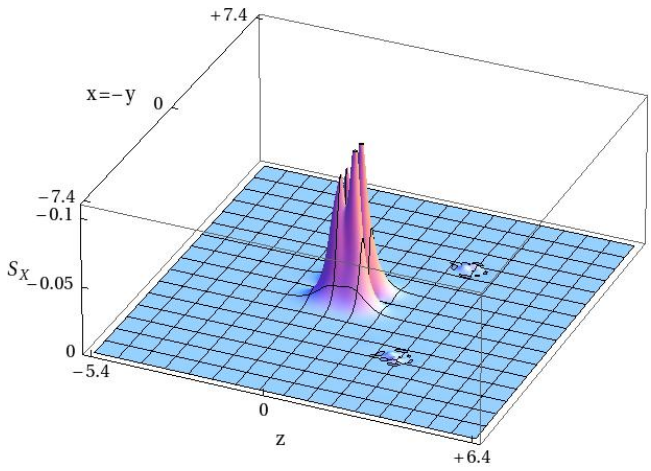

(a-data, $\mathrm{ClCoCl}$ small angle plane)

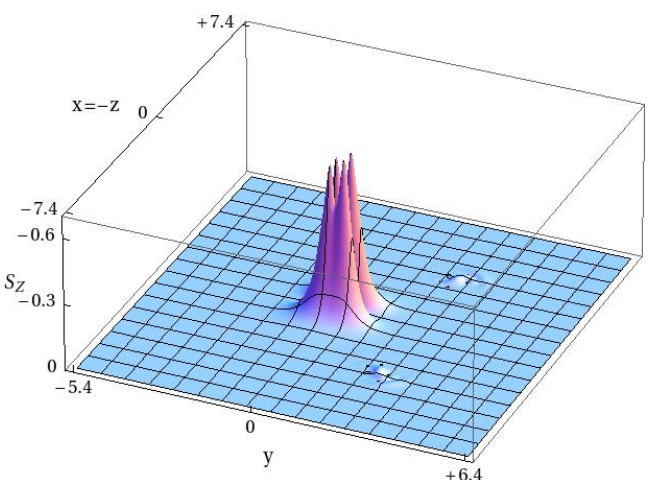

(c-data, $\mathrm{ClCoCl}$ large angle plane)

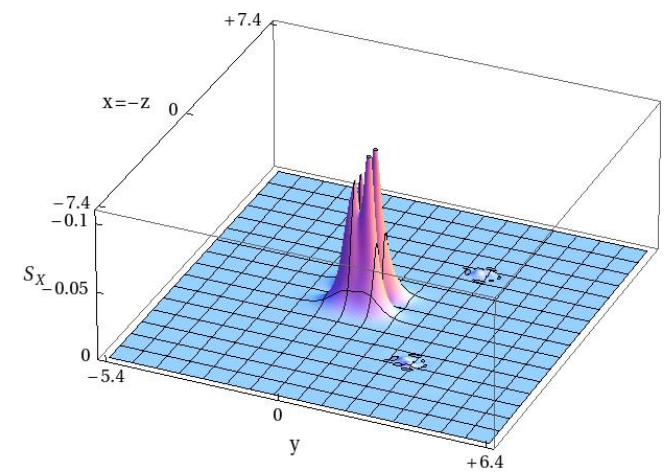

(a-data, $\mathrm{ClCoCl}$ large angle plane)

Fig. 4. Component aligned with neutron polarization of the spin density of $\mathrm{CoCl}_{4}^{2-}$ in atomic units. See Fig. 1 for the definition of the planes. 


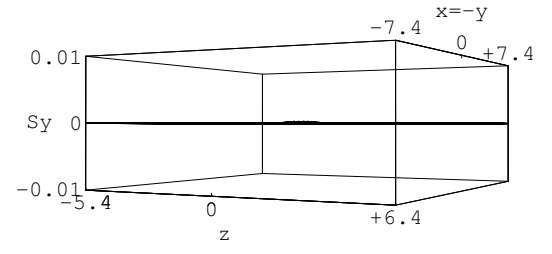

(a-data, $\mathrm{ClCoCl}$ small angle plane)

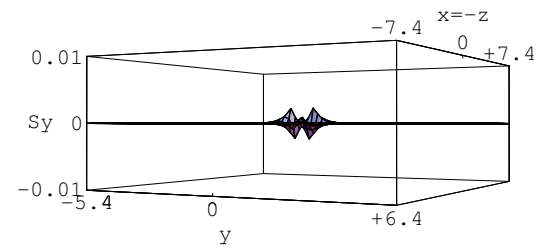

(a-data, $\mathrm{ClCoCl}$ large angle plane)

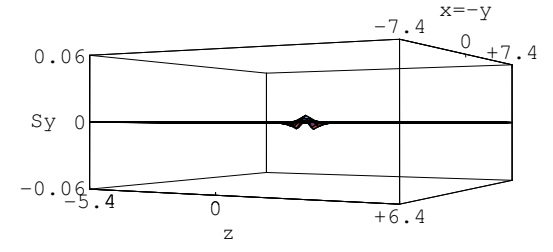

(c-data, $\mathrm{ClCoCl}$ small angle plane)

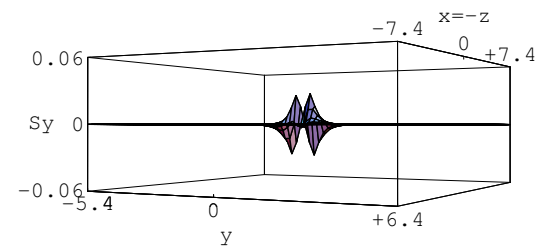

(c-data, $\mathrm{ClCoCl}$ large angle plane)

Fig. 5. y-component of the spin density of $\mathrm{CoCl}_{4}^{2-}$ from optimized density operators. The figures on the left-hand side correspond to the density operator optimized on the experimental a-data set with an applied magnetic field along the $\mathrm{Cs}_{3} \mathrm{CoCl}_{5}$ crystal a-axis. The figures on the right-hand side correspond to the density operator optimized on the experimental c-data set with an applied magnetic field along the $\mathrm{Cs}_{3} \mathrm{CoCl}_{5}$ crystal c-axis. See Fig. 1 for the definition of the planes. 


\section{References}

[1] P. Cassam-Chenaï, J. Chem. Phys., 2002, 116, 8677-8690.

[2] P. Cassam-Chenaï, S.K. Wolff et G.S. Chandler, Int. J. Quantum Chem., 1996, 60, 667.

[3] S.-Y. Wu, H.-N. Dong, X.-Y. Gao, Spectr. Acta, 2006, A63, 749.

[4] T. Qu, X.-Y. Kuang, Y.-F. Li, R.-P. Chai, Chem. Phys. Lett., 2011, 504, 170.

[5] M. C. Caputo, P. Lazzeretti, Chem. Phys., 2003, 288, 281-289.

[6] M. C. Pelloni, P. Lazzeretti, J. Chem. Phys., 2012, 136, 164110.

[7] G. S. Chandler, B. N. Figgis, R. A. Phillips, P. A. Reynolds, R. Mason, G. A. Williams, Proc. Royal Soc. London, 1982, A 384, 31.

[8] P. Cassam-Chenaï, Int. J. Quantum Chem., 2001, 81, 11.

[9] Wolfram Research, Inc., Mathematica, Version 8.0, Champaign, IL (2010).

[10] D. Jayatilaka and D. J. Grimwood, in Computational Science - ICCS 2003, (Lectures Notes in Computer Sciences Vol. 2660, Sloot P., Abramson D., Bogdanov A., Gorbachev Y., Dongarra J., and Zomaya A. editors, Springer, Berlin and Heidelberg, 2003) p.142-151.

[11] A. Soncini, J. Chem. Theory and Computation, 2007, 3, 2243-2257.

[12] P. Cassam-Chenaï, Int. J. Quantum Chem., 1995, 54, 201.

[13] R. P. Van Stapele, H. G. Beljers, P. F. Bongers, and H. Zijlstra, J. Chem. Phys., 1966, 44, 3719 .

[14] H. B. Schlegel, J. S. Binkley, J. A. Pople, J. Chem. Phys., 1984, 80, 1976.

[15] H. F. King, M. Dupuis, J. Chem. Phys., 1976, 65, 111.

[16] H. F. King, M. Dupuis, J. Comp. Phys., 1976, 21, 144.

[17] R. P. Sagar, V. H. Smith Jr., Int. J. Quantum Chem., 1992, 42, 827.

[18] W. H. Press, S. A. Teukolsky, W. T. Vetterling and B. P. Flannery, "Numerical Recipes 3rd Edition: The Art of Scientific Computing", (Cambridge University Press, New York, 2007). 
[19] M. Hossein, Master Thesis, 2006, Cartographie du champ magnètique généré par une molécule, University of Nice - Sophia Antipolis. 